\title{
Realization and Modeling of Metamaterials Made of rf Superconducting Quantum-Interference Devices
}

\author{
M. Trepanier, ${ }^{1, *}$ Daimeng Zhang, ${ }^{2, \dagger}$ Oleg Mukhanov, ${ }^{3}$ and Steven M. Anlage ${ }^{1,2}$ \\ ${ }^{1}$ Department of Physics, CNAM, University of Maryland, College Park, Maryland 20742-4111, USA \\ ${ }^{2}$ Department of Electrical and Computer Engineering, University of Maryland, College Park, Maryland 20742-3285, USA \\ ${ }^{3}$ Hypres, Inc., 175 Clearbrook Road, Elmsford, New York 10523, USA
}

(Received 26 July 2013; revised manuscript received 22 October 2013; published 18 December 2013)

\begin{abstract}
We have prepared meta-atoms based on radio-frequency superconducting quantum-interference devices (rf SQUIDs) and examined their tunability with dc magnetic field, rf current, and temperature. rf SQUIDs are superconducting split-ring resonators in which the usual capacitance is supplemented with a Josephson junction, which introduces strong nonlinearity in the rf properties. We find excellent agreement between the data and a model that regards the Josephson junction as the resistively and capacitively shunted junction. A magnetic field tunability of $80 \mathrm{THz} / \mathrm{G}$ at $12 \mathrm{GHz}$ is observed, a total tunability of $56 \%$ is achieved, and a unique electromagnetically induced transparency feature at intermediate excitation powers is demonstrated for the first time. An rf SQUID metamaterial is shown to have qualitatively the same behavior as a single rf SQUID with regard to dc flux and temperature tuning.
\end{abstract}

DOI: 10.1103/PhysRevX.3.041029

\section{INTRODUCTION}

Metamaterials are artificially structured media, designed to have electromagnetic properties not found in nature. These properties arise from both the structure of individual meta-atoms and the interactions between them, resulting in interesting collective behavior. A wide variety of properties and applications has been pursued using metamaterials, including a negative index of refraction [1-3], superresolution imaging [4,5], cloaking [6,7], transformation optics [8,9], and perfect absorption [10].

Most of the applications invoking novel optical properties impose three stringent constraints. First, the metamaterial must have low attenuation. Features such as evanescent wave amplification [11] and negative refraction are strongly suppressed by even small amounts of loss [11-16]. For example, the enhanced loss in metamaterials approaching the plasmonic limit has imposed a severe limitation on visible-wavelength metamaterials composed of noble-metal nanostructures [17-20]. Second, the metaatoms must be of deep subwavelength dimensions to achieve the metamaterial limit, as opposed to the photonic crystal limit. This constraint has been an issue in both the visible and microwave regimes, where meta-atom sizes often approach the scale of the wavelength to minimize losses [21,22]. Third, it is desirable to make metamaterials that have textured properties in space (e.g., for cloaking

\footnotetext{
*mctrep@umd.edu

†dmchang@umd.edu
}

Published by the American Physical Society under the terms of the Creative Commons Attribution 3.0 License. Further distribution of this work must maintain attribution to the author(s) and the published article's title, journal citation, and DOI.
Subject Areas: Condensed Matter Physics, Metamaterials, Superconductivity

or transformation optics) or that can be tuned and reconfigured after the metamaterial has been fabricated [23]. The ability to tune the electromagnetic response over a wide range, and on short time scales, is desirable for applications such as software-defined radio [24] and filters for digital rf receivers [25,26]. Superconducting metamaterials have been proposed to address all three of these constraints [27]. In addition, the quantum coherent nature of the superconducting state leads to qualitatively new phenomena such as fluxoid quantization and Josephson effects. Here, we focus on the tunability and reconfigurable nature of superconducting Josephson metamaterials.

\section{A. Tunable superconducting metamaterials}

Superconductors are fundamentally nonlinear due to the nonlinear Meissner effect [28,29]. In general, this intrinsic nonlinearity will be encountered near the limits of parameter space spanned by temperature, applied magnetic field, and applied current.

Superconducting metamaterials are tunable with temperature because of the temperature dependence of the superfluid density and kinetic inductance [30,31]. This type of tunability has been exploited in various superconducting metamaterials with some success [32-36]. However, temperature tuning is slow since the thermal inertia of the meta-atoms can be large, even at low temperatures. Typical estimates for temperature-tuning response times are on the order of $10 \mu \mathrm{s}$ [37].

Applied currents can also be used to tune superconducting metamaterials; they can cause superfluid depairing, which increases the kinetic inductance [38]. For example, applied currents can tune the sub- $\mathrm{THz}$ transmission of a metamaterial composed of a network of resonators connected by a superconducting wire loop [37]. However, 
applied currents often induce magnetic vortices in the superconductor before the depairing critical current is reached $[37,39]$. These vortices move under the influence of the high-frequency currents, creating enhanced inductance and dissipation. The additional dissipation renders the superconductors less attractive for applications.

Magnetic field tuning of superconductors is attractive for applications because it can have a large effect on metamaterial properties. For example, superconducting split-ring resonators (SRRs) have been tuned by both dc and rf magnetic fields [40,41]. A dc magnetic field was shown to add magnetic flux to a thin-film $\mathrm{Nb}$ SRR, increasing its inductance and loss [40]. The rf magnetic field created enhanced rf screening currents at discrete locations in the SRR, resulting in enhanced inductance and dissipation as magnetic flux moved into and out of the superconducting film at high frequency $[40,42-46]$. It was also found that a superconducting resonator exhibiting an analogue of electromagnetically induced transparency showed a strong switching behavior at high excitation power [36], for similar reasons. However, the insertion of magnetic flux into superconducting materials is often too slow and too dissipative for tuning applications; a low-dissipation quantum effect would be better. For example, flux quantization could be used to discretely tune a superconducting meta-atom [47]. The addition of the Josephson effect to superconducting metamaterials adds a mechanism of tunability that offers a large degree of high-speed tunability and low dissipation.

\section{B. Superconducting meta-atoms employing the Josephson effect}

A superconductor can be described by a macroscopic phase-coherent complex quantum wave function $\Psi=$ $\sqrt{n_{s}} e^{i \theta}$ [48]. This wave function inherits its phase coherence from the underlying microscopic BCS wave function describing the Cooper pairing of electrons in the metal. The absolute square of the macroscopic quantum wave function is the local superfluid density $n_{s} \sim|\Psi|^{2}$ [49]. When two superconductors are brought close together and separated by a thin insulating barrier, there can be tunneling of Cooper pairs between the two materials $[48,50]$. This tunneling results in two types of Josephson effect.

The dc Josephson effect produces a dc current between the two superconductors that depends on the phase difference between their macroscopic quantum wave functions as $I=I_{c} \sin \delta(t)$, where $\delta(t)=\theta_{1}(t)-\theta_{2}(t)-\frac{2 \pi}{\Phi_{0}} \int_{1}^{2} \vec{A}(\vec{r}, t) \cdot d \vec{l}$ is the gauge-invariant phase difference between superconductors 1 and $2, \vec{A}(\vec{r}, t)$ is the magnetic vector potential in the region between the superconductors, $I_{c}$ is the critical (maximum) current of the junction, and $\Phi_{0}=\frac{h}{2 e} \cong$ $2.07 \times 10^{-15} \mathrm{Tm}^{2}$ is the flux quantum (where $h$ is Planck's constant and $e$ is the electronic charge).

The ac Josephson effect relates a dc voltage drop across the junction to a time-varying gauge-invariant phase difference and therefore to an ac current in the junction: $\frac{d \delta}{d t}=\frac{2 \pi}{\Phi_{0}} V$, where $V$ is the dc voltage across the junction. In general, the ac impedance of a Josephson junction contains both resistive and reactive components [51].

In the presence of both dc and ac currents, the inductance of the junction can be described approximately as [49]

$$
L_{\mathrm{JJ}}=\frac{\Phi_{0}}{2 \pi I_{c} \cos \delta} .
$$

The Josephson inductance is a strong function of applied currents and fields. The tuning of Josephson inductance in a transmission-line metamaterial geometry was considered theoretically by Salehi, Majedi, and Mansour [52,53].

\section{SQUID metamaterials}

In this work, we focus on meta-atoms comprised of a superconducting loop interrupted by a single Josephson junction, commonly known as a radio-frequency superconducting quantum-interference device (rf SQUID). Since the rf SQUID meta-atom [shown in Fig. 1(b)] is a natural quantum analogue of the SRR, we examine

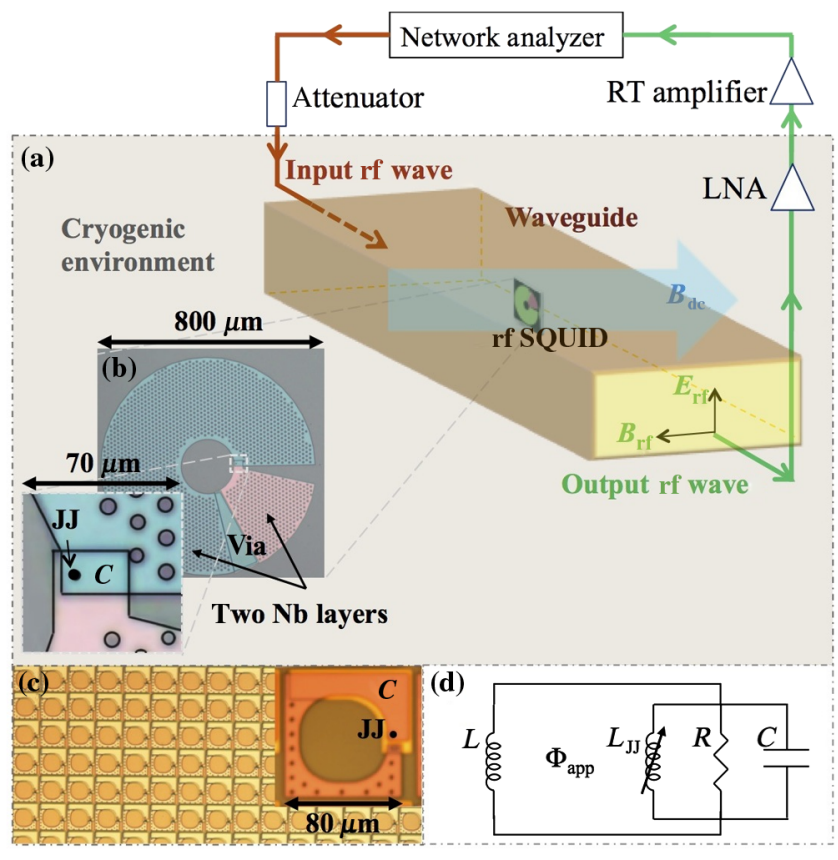

FIG. 1. (a) Schematic diagram of the experiment showing the flow of rf signal from the network analyzer (at RT), through the attenuator, into the waveguide (in the cryogenic environment), through the rf SQUID biased by dc magnetic field, out of the waveguide through a series of amplifiers, and back to the network analyzer. (b) Micrograph of an rf SQUID meta-atom, consisting of two perforated $\mathrm{Nb}$ layers connected by a via and a Josephson junction (JJ). The overlap capacitance is the small rectangular region immediately surrounding the junction. (c) Micrograph of a portion of the $27 \times 27 \mathrm{rf} \mathrm{SQUID}$ array and a single meta-atom that composes the array. (d) Circuit diagram of the rf SQUID modeled as an RCSJ in parallel with loop inductance $L$. 
the electromagnetic properties of the SQUID near its selfresonant frequency.

The original purpose of the rf SQUID was to measure small magnetic fields and operate as a flux-to-frequency transducer [54,55]. The first proposal to use an array of rf SQUIDs as a metamaterial was made by $\mathrm{Du}, \mathrm{Chen}$, and $\mathrm{Li}$ $[56,57]$. Their calculation assumes that the SQUID has quantized energy levels and considers the interaction of microwave photons with the lowest states of the SQUID potential. For small detuning of the photon frequency above the transition from the ground state to the first excited state, the medium presents a negative effective permeability. The frequency region of negative permeability is diminished by a nonzero dephasing rate, and negative permeability will disappear for dephasing rates larger than a critical value.

rf SQUIDs interacting with classical electromagnetic fields were modeled by Lazarides and Tsironis [58]. They considered a two-dimensional array of rf SQUIDs in which the Josephson junction was treated as a parallel combination of resistance, capacitance, and Josephson inductance. Near resonance, a single rf SQUID can have a large diamagnetic response. An rf SQUID array displays a negative real part of effective permeability for a range of frequencies and magnetic fields. The permeability is oscillatory as a function of applied magnetic flux and is suppressed with applied fields that induce currents in excess of the critical current of the Josephson junction. Similar calculations, which included interactions between the rf SQUIDs, were carried out by Maimistov and Gabitov [59]. The response of a two-dimensional rf SQUID metamaterial under an applied oscillating magnetic field was also numerically investigated by Lazarides and Tsironis [60]. The weakly coupled elements of the metamaterial are predicted to show bistability and synchronization.

Related work on a one-dimensional array of superconducting islands that can act as quantum bits (qubits) was considered by Rakhmanov et al. [61]. When interacting with classical electromagnetic radiation, the array can create a quantum photonic crystal, which can support a variety of nonlinear wave excitations. A similar idea based on a SQUID transmission line was implemented to perform parametric amplification of microwave signals $[62,63]$. A one-dimensional array of dc SQUIDs has been utilized as a tunable composite right- or left-handed transmission line [64]. A dc field-tunable one-dimensional SQUID metamaterial embedded in a coplanar waveguide structure has recently been demonstrated [65-67].

It is expected that SQUID metamaterials will be able to perform functions similar to galvanically connected SQUID arrays that have been developed in recent years. One possible application takes advantage of the SQUID's extreme sensitivity to magnetic flux to create compact, wideband antennas, sensitive to high-frequency magnetic fields, as opposed to electric fields [68-71]. Other possible applications include low noise amplifiers for rf sensing and qubit readout $[63,72,73]$ and highly sensitive magnetometers and filters [74-76].

Our objective in this paper is to demonstrate the first SQUID metamaterial in a geometry that can be easily extended to a fully three-dimensional structure that can interact with free-space electromagnetic waves. We demonstrate that rf SQUID meta-atoms are remarkably tunable with very small dc magnetic fields, as well as temperature and rf currents.

The remainder of the paper is organized as follows. We first present models of rf SQUID linear and nonlinear behavior. Then, we discuss the design and fabrication of a single rf SQUID meta-atom and a dense array of such SQUIDs. Section V presents our experimental results of the rf SQUID meta-atom and array in waveguide geometries, demonstrating tunability of the meta-atom properties with dc flux, temperature, and rf flux. Section VI is a comparison of data and model predictions and a discussion of the results, and Sec. VII serves as a conclusion.

\section{MODELING}

An ideal Josephson junction can be treated as an inductor with a variable inductance given by Eq. (1). A more realistic model (valid for low frequencies and currents) shunts the junction with a subgap resistance $R$ (representing the tunneling of normal-state electrons across the junction) and capacitance $C$ (the capacitance of two overlapping conductors separated by an insulator) [49]. We model the rf SQUID as a resistively and capacitively shunted junction (RCSJ) in parallel with an inductor representing the inductance $L$ of the superconducting loop. The result is an $R L C$ circuit [see the circuit diagram in Fig. 1(d)] with a resonant frequency given by

$$
f_{0}\left(T, \Phi_{\text {app }}\right)=\frac{1}{\left.2 \pi \sqrt{\left(\frac{1}{L}+\frac{1}{L_{\mathrm{JJ}}\left(T, \Phi_{\mathrm{app}}\right.}\right)}\right)^{-1} C},
$$

where $T$ is the temperature and $\Phi_{\text {app }}$ is the magnetic flux applied to the SQUID loop. The quality factor for this parallel $R L C$ circuit is proportional to the resonant frequency and given by

$$
Q=R \sqrt{\frac{C}{\left(\frac{1}{L}+\frac{1}{L_{\mathrm{JJ}}\left(T, \Phi_{\text {app }}\right.}\right)^{-1}}}=2 \pi R C f_{0}\left(T, \Phi_{\mathrm{app}}\right) .
$$

To understand the behavior of the resonance as a function of temperature and applied flux, it is necessary to solve for the time-dependent gauge-invariant phase difference $\delta\left(T, \Phi_{\text {app }}\right)$.

We use the RCSJ model to solve for $\delta$ subject to the condition that the total flux through the loop must be an integer number $(n)$ of flux quanta, i.e., $\Phi=n \Phi_{0}$. The total flux $\Phi$ is related to the applied flux $\Phi_{\text {app }}$ and the currentinduced flux by

$$
\Phi_{\mathrm{app}}=\Phi+L I
$$


where $I$ is the current through the loop, which is the sum of the currents through the junction, the resistor, and the capacitor. We can rewrite Eq. (4) in the terms of $\delta$ as

$$
\begin{aligned}
\Phi_{\mathrm{dc}} & +\Phi_{\mathrm{rf}} \sin \omega t \\
& =\frac{\Phi_{0} \delta}{2 \pi}+L\left(I_{c} \sin \delta+\frac{1}{R} \frac{\Phi_{0}}{2 \pi} \frac{d \delta}{d t}+C \frac{\Phi_{0}}{2 \pi} \frac{d^{2} \delta}{d t^{2}}\right),
\end{aligned}
$$

where $\Phi_{\mathrm{dc}}$ is the dc flux bias of the applied magnetic field and $\Phi_{\mathrm{rf}}$ and $\omega$ are the amplitude and angular frequency of the applied rf field, respectively. By making the substitution $\Phi=\frac{\Phi_{0} \delta}{2 \pi}$, we have taken $n=1$ because the choice of integer shifts $\delta$ (which has $2 \pi$ periodicity) by $2 \pi$. Equation (5) can be recast in nondimensional form as

$2 \pi\left[f_{\mathrm{dc}}+f_{\mathrm{rf}} \sin \Omega \tau\right]=\delta+\beta_{\mathrm{rf}} \sin \delta+\frac{1}{Q_{\mathrm{geo}}} \frac{d \delta}{d \tau}+\frac{d^{2} \delta}{d \tau^{2}}$,

where $\omega_{0, \text { geo }}=\sqrt{\frac{1}{L C}}, \tau=\omega_{0, \text { geo }} t, \Omega=\frac{\omega}{\omega_{0, \text { geo }}}, \beta_{\mathrm{rf}}=\frac{2 \pi L I_{c}}{\Phi_{0}}$, $Q_{\mathrm{geo}}=R \sqrt{\frac{C}{L}}, f_{\mathrm{rf}}=\frac{\Phi_{\mathrm{rf}}}{\Phi_{0}}$, and $f_{\mathrm{dc}}=\frac{\Phi_{\mathrm{dc}}}{\Phi_{0}}$.

A well-studied mechanical analogue of the Josephson junction is the driven and damped pendulum [77]. Equation (6) resembles the nonlinear differential equation that governs that system. However, incorporating the junction into a superconducting loop that is subject to flux quantization introduces an additional term that is linear in $\delta$ on the right-hand side of Eq. (6). Unlike the driven, damped pendulum, this equation is linear in the high power limit in addition to the low power limit. For our chosen parameters, $\delta$ is always periodic in time and its variation is dominated by the same frequency as the driving flux. We do not observe chaos for parameter values relevant to the experiments discussed below.

At intermediate powers, the full nonlinear Eq. (6) must be solved for $\delta$, and therefore $f_{0}\left(T, f_{\mathrm{dc}}, f_{\mathrm{rf}}\right)$, but the equation can be linearized and simplified in the low and high power limits. In the low rf power limit [where it is assumed that the time-varying component of the gauge-invariant phase difference is very small, i.e., $\delta_{\mathrm{rf}}(\tau) \ll 1$ ], Eq. (6) can be linearized by separating the phase difference into dc and rf components, i.e.,

$$
\delta=\delta_{\mathrm{dc}}+\delta_{\mathrm{rf}}(\tau) .
$$

Equation (6) simplifies to the following time-independent and time-dependent equations:

$$
\begin{gathered}
2 \pi f_{\mathrm{dc}}=\delta_{\mathrm{dc}}+\beta_{\mathrm{rf}} \sin \delta_{\mathrm{dc}}, \\
2 \pi f_{\mathrm{rf}} \sin \Omega \tau=\alpha \delta_{\mathrm{rf}}+\frac{1}{Q_{\mathrm{geo}}} \frac{d \delta_{\mathrm{rf}}}{d \tau}+\frac{d^{2} \delta_{\mathrm{rf}}}{d \tau^{2}},
\end{gathered}
$$

where $\alpha=1+\beta_{\mathrm{rf}} \cos \delta_{\mathrm{dc}}$. In this case, the dc flux dictates $\delta_{\mathrm{dc}}$ and Eq. (9) has an analytic solution for $\delta_{\mathrm{rf}}$ given by

$$
\delta_{\mathrm{rf}}=2 \pi f_{\mathrm{rf}} \frac{\left(\alpha-\Omega^{2}\right) \sin \Omega \tau-\left(\Omega / Q_{\mathrm{geo}}\right) \cos \Omega \tau}{\left(\alpha-\Omega^{2}\right)^{2}+\left(\Omega / Q_{\mathrm{geo}}\right)^{2}} .
$$

From this result, one finds that $\delta$ and consequently $f_{0}\left(T, f_{\mathrm{dc}}\right)$ are periodic functions of dc flux $f_{\mathrm{dc}}$, with maximum and minimum values at $f_{\mathrm{dc}}=n$ and $f_{\mathrm{dc}}=n+1 / 2$, respectively, where $n$ is any integer.

The resonant frequency of the SQUID $f_{0}$ is also temperature dependent because of the temperature dependence of the critical current $I_{c}$. As temperature increases, the maximum frequency decreases and the minimum frequency increases, resulting in a reduction of the total flux tunability of the SQUID. When the temperature reaches the critical temperature of niobium $\left(T_{c}=9.2 \mathrm{~K}\right),\left|L_{\mathrm{JJ}}\right|$ is expected to diverge (since $I_{c} \rightarrow 0$ ). In this case, the Josephson junction loses the ability to modify the resonance and the resonant frequency reduces to

$$
f_{0, \text { geo }}=\frac{1}{2 \pi \sqrt{L C}} .
$$

This resonance depends solely on the nonjunction properties of the SQUID and is insensitive to applied flux $\Phi_{\text {app }}$ and temperature $T$.

Equation (6) is also linear in the high power limit (where it is assumed that $\delta_{\mathrm{rf}} \gg 1$ since $\beta_{\mathrm{rf}} \sin \delta+\delta \approx \delta$ for $\beta_{\mathrm{rf}}<1$ ), where it separates into the following timedependent and time-independent equations:

$$
\begin{gathered}
2 \pi f_{\mathrm{dc}}=\delta_{\mathrm{dc}}, \\
2 \pi f_{\mathrm{rf}} \sin (\Omega \tau)=\delta_{\mathrm{rf}}+\frac{1}{Q_{\mathrm{geo}}} \frac{d \delta_{\mathrm{rf}}}{d \tau}+\frac{d^{2} \delta_{\mathrm{rf}}}{d \tau^{2}} .
\end{gathered}
$$

Equation (13) has the same analytic solution as Eq. (10) with $\alpha$ replaced by 1 , so unlike Eq. (10), it has no dependence on temperature or dc flux. In the high power limit, the resonant frequency reduces to the same value as in the high temperature limit; see Eq. (11).

\section{DESIGN AND FABRICATION}

We have designed and measured both a single rf SQUID meta-atom and a dense $27 \times 27$ array of meta-atoms, which were manufactured using the Hypres $0.3-\mu \mathrm{A} / \mu \mathrm{m}^{2} \mathrm{Nb} / \mathrm{AlO}_{x} / \mathrm{Nb}$ junction process on silicon substrates. However, measurements of other junctions from this run suggest that the critical current density is closer to $0.2 \mu \mathrm{A} / \mu \mathrm{m}^{2}$ at $4.2 \mathrm{~K}$. The superconducting loop is composed of two $\mathrm{Nb}$ films (135 and $300 \mathrm{~nm}$ thick) that are connected by a via and a $\mathrm{Nb} / \mathrm{AlO}_{x} / \mathrm{Nb}$ Josephson junction [see Fig. 1(b)]. There is additional capacitance where these layers overlap (with $\mathrm{SiO}_{2}$ dielectric) that is necessary to bring the resonant frequency within the measurable range. When designing the SQUIDs, we have control over the inner and outer radii of the loop and thus the loop inductance $L$, the critical current of the junction $I_{c}$, and the overlap capacitance $C$. Values for $L, I_{c}$, and $C$ are chosen to maximize tunability within the measurable frequency range $6.5-22 \mathrm{GHz}$ (dictated by the available 
waveguides) while remaining in the low noise $[\Gamma=$ $\frac{2 \pi k_{B} T}{\Phi_{0} I_{c}}<1$ and $\left.L_{F}=\frac{1}{k_{B} T}\left(\frac{\Phi_{0}}{2 \pi}\right)^{2} \gg L[78]\right]$ and nonhysteretic $\left(\beta_{\mathrm{rf}}=\frac{2 \pi L I_{c}}{\Phi_{0}}<1\right)$ limits.

For the single meta-atom shown in Fig. 1(b), the inner and outer radii of the SQUID loop are 100 and $400 \mu \mathrm{m}$, respectively, with 3 - $\mu$ m-diameter holes in the film every $10 \mu \mathrm{m}$ to pin vortices [79]. We estimate $L$ to be $0.33 \mathrm{nH}$ based on FastHenry calculations [80]. The area of the Josephson junction and the area of the capacitor are 5.3 and $600 \mu \mathrm{m}^{2}$, respectively, yielding nominal design values of $I_{c}(4.2 \mathrm{~K})=0.97 \mu \mathrm{A}$ and $C=0.32 \mathrm{pF}$.

Figure $1(\mathrm{c})$ shows a portion of a $27 \times 27$ array of nominally identical rf SQUID meta-atoms with a centerto-center separation of $83 \mu \mathrm{m}$. The inner and outer radii of the SQUID loops are 30 and $40 \mu \mathrm{m}$, respectively. We estimate $L$ to be $0.12 \mathrm{nH}$. The area of the Josephson junction and the area of the capacitor are 13 and $1800 \mu \mathrm{m}^{2}$, respectively, yielding nominal design values of $I_{c}(4.2 \mathrm{~K})=3.7 \mu \mathrm{A}$ and $C=0.84 \mathrm{pF}$.

\section{EXPERIMENT}

The single rf SQUID (and later the $27 \times 27 \mathrm{rf}$ SQUID array) is oriented in a 7.6-cm-long $\mathrm{Ku}(\mathrm{K})$ rectangular waveguide that has a single propagating mode operating frequency range from 9.5 to $19 \mathrm{GHz}$ (15 to $26 \mathrm{GHz}$ ), as shown in Fig. 1(a). An attenuated microwave signal from an Agilent E8364C network analyzer excites a TE ${ }_{10}$ (transverse electric) mode in the waveguide, producing an $\mathrm{rf}$ magnetic field $B_{\text {rf }}$ perpendicular to the plane of the SQUID. The transmission $S_{21}$ is measured after a cryogenic low noise amplifier (LNA) and a room-temperature (RT) amplifier. The experiment is conducted in a three-stage pulsed-tube cryostat with a sample base temperature of $6.5 \mathrm{~K}$. The sample is magnetically shielded by a $\mu$-metal cylinder and a superconducting niobium open cylinder inside the cryostat. Superconducting coils surrounding the waveguide bias the SQUID by generating a perpendicular dc magnetic field $B_{\mathrm{dc}}$ in the range from 0 to $\pm 1 \mu \mathrm{T}$.

The resonance is detected as a dip in the frequencydependent transmission magnitude through the waveguide $\left|S_{21}(\omega)\right|$. We have considered two methods for calculating scattering parameters from the solution for $\delta(t)$. One method is to consider the SQUID an effective medium with an effective relative permeability $\mu_{r}[65,81]$

$$
\mu_{r}=1+F\left(\left\langle\frac{\Phi_{\mathrm{ac}}}{\Phi_{\mathrm{rf}} \sin \omega t}\right\rangle-1\right)
$$

where $\Phi_{\mathrm{ac}}$ is the ac flux response of the loop, the angled brackets represent time averaging, and $F$ is the filling fraction of the SQUID in the medium [57,58]. $S_{21}$ is proportional to the ratio of the transmitted electric field $E_{T}$ to the incident field $E_{0}$, and can be calculated using the continuity of $E$ and $H$ fields at the boundaries of the effective medium and the empty waveguide as

$$
S_{21}=\sqrt{\gamma} \frac{E_{T}}{E_{0}}=\frac{\sqrt{\gamma}}{\cos k l-\frac{i}{2}\left(\frac{1}{\gamma}+\gamma\right) \sin k l}
$$

where $l$ is the length of the medium, $\gamma=\frac{k}{\mu_{r} k_{0}}, k=$ $\sqrt{\mu_{r}\left(\frac{\omega}{c}\right)^{2}-\left(\frac{\pi}{a}\right)^{2}}$ is the wave number in the medium, and $k_{0}=k\left(\mu_{r}=1\right)$ is the wave number in the empty waveguide. For the single SQUID (as opposed to the array), the choice of $l$ and $F$ is not straightforward. They are used as fitting parameters (along with resistance $R$ ) for the width and depth of the measured resonances. The important parameter for the fit is the relationship between $F$ and $l$, which we find to be $F=\frac{A_{\text {loop }}}{0.79 l}$, where $A_{\text {loop }}$ is the area of the SQUID loop (calculated from the average radius).

An alternate method for estimating $S_{21}$ focuses on the power dissipated in the resistor $R$ in Fig. 1(d):

$$
S_{21}=\sqrt{\frac{P_{T}}{P_{0}}}=\sqrt{1-\frac{V^{2} / R}{P_{0}}},
$$

where $P_{T}$ is the transmitted power and $P_{0}$ is the incident power. This method assumes that the only power not transmitted through the waveguide is the power dissipated in the resistor. It does not account for reflection or other loss mechanisms.

\section{RESULTS}

The resonance of an $\mathrm{rf}$ SQUID responds to three tuning parameters: dc magnetic field, temperature, and rf power. Modifying the resonance with dc magnetic field is the most straightforward. Figure 2 shows the experimental results for $\left|S_{21}\right|$ of an rf SQUID meta-atom as a function of dc flux and frequency. The incident $\mathrm{rf}$ power and the temperature are fixed at $-80 \mathrm{dBm}$ and $6.5 \mathrm{~K}$, respectively. Resonance dips in $\left|S_{21}(\omega)\right|$ appear as the red features against a yellow background of unaffected signals $\left(\left|S_{21}\right|=0 \mathrm{~dB}\right)$. The signal is extracted by subtracting $\left|S_{21}\right|$ at $16 \mathrm{~K}$ (which is well above the critical temperature) from $\left|S_{21}\right|$ at $6.5 \mathrm{~K}$, removing a background variation, and applying a threshold to identify the resonance. The resonance shows good periodicity with de flux, with a maximum value of $16.9 \pm$ $0.3 \mathrm{GHz}$ and a minimum at or below $10 \mathrm{GHz}$. The cutoff frequency of the $\mathrm{Ku}$ waveguide is $9.5 \mathrm{GHz}$, which imposes a lower frequency limit on this measurement. The same sample measured in an $X$-band waveguide (single propagating mode operating frequency range from 6.6 to $13 \mathrm{GHz}$ ) has a minimum resonant frequency of $9.5 \pm$ $0.5 \mathrm{GHz}$.

The small magnitude of the resonance dips can be attributed to the small size of the SQUID relative to the waveguide. The shallow resonance dips become less of a factor when considering arrays of SQUIDs that occupy a more appreciable fraction of the waveguide cross section. The quality factor $Q$ is extracted by fitting the $\left|S_{21}(\omega)\right|$ data to a model of an $R L C$ resonator inductively coupled to a 


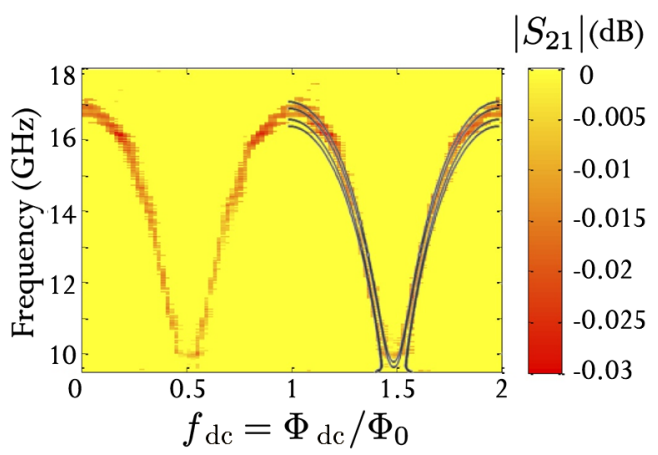

FIG. 2. $\left|S_{21}\right|$ of a single rf SQUID meta-atom as a function of frequency and applied dc flux at $-80-\mathrm{dBm}$ rf power and $6.5 \mathrm{~K}$. The resonant response is identified by the red features. There are contour lines for $\left|S_{21}\right|$ at -0.005 and $-0.01 \mathrm{~dB}$ generated by the model; see Eq. (15).

transmission line [82]. $Q$ also shows periodicity with dc flux, following the same trend as the resonant frequency [Eq. (3)], with a maximum value of 54 and a minimum at or below 20.

At an rf power of $-80 \mathrm{dBm}\left(\Phi_{\mathrm{rf}} \approx 0.003 \Phi_{0}\right)$, the low power linearized equation [Eq. (9)] is valid (since $\delta_{\text {rf }} \ll 1$ ). Treating the rf SQUID as an effective medium in the waveguide, $\left|S_{21}\left(\omega, \Phi_{\mathrm{dc}}\right)\right|$ is calculated from Eqs. (8), (10), (14), and (15) and plotted as the contour lines in Fig. 2. The capacitance $C$ and critical current $I_{c}(6.5 \mathrm{~K})$ are adjusted from their nominal values and taken to be $0.42 \mathrm{pF}$ and $0.55 \mu \mathrm{A}$, respectively. These values are chosen so that the resonant frequency at $T=6.5 \mathrm{~K}$ and $\Phi_{\mathrm{dc}}=0$ agrees with the measurements in both the low and high power limits, i.e., $f_{0}\left(6.5 \mathrm{~K}, \Phi_{\mathrm{dc}}=0\right)=16.8 \mathrm{GHz}$ for low power [Eq. (2)] and $f_{0, \text { geo }}=13.5 \mathrm{GHz}$ for high power [Eq. (11)]. The model and the data agree both in terms of the flux dependence and magnitude of the tunability (approximately $7 \mathrm{GHz}$ ), and in terms of the magnitude of the resonance dip.

The dc flux tunability is modified by changes in temperature. The red features in Fig. 3 show the dc flux-tuned resonance at $6.5,7.6$, and $8.3 \mathrm{~K}$ for an $\mathrm{rf}$ power of $-80 \mathrm{dBm}$. The flux tunability is reduced from $7 \mathrm{GHz}$ at $6.5 \mathrm{~K}$ to $3 \mathrm{GHz}$ at $7.6 \mathrm{~K}$ and $1 \mathrm{GHz}$ at $8.3 \mathrm{~K}$. The quality factor $Q$ is 50 at $\Phi_{\mathrm{dc}}=0$ and does not show significant variation with temperature. The gray lines follow the resonance by using the solution for $\delta_{\mathrm{dc}}$ from Eq. (8) and calculating the resonant frequency using Eqs. (1) and (2) (assuming $\delta \approx \delta_{\mathrm{dc}}$ ). The reduction in tunability with increased temperature is consistent with the model. The only parameter varied to fit these data is the temperaturedependent critical current.

We also measure $\left|S_{21}\right|$ as a function of frequency and dc flux at low power for various fixed temperatures ranging from 6.5 to $9.2 \mathrm{~K}$ (not shown). For $f_{\mathrm{dc}}=0$, the resonant frequency decreases from 17 to $13.5 \mathrm{GHz}$ with increasing temperature, and for $f_{\mathrm{dc}}=1 / 2$, it increases from 9.5 to 13.5 GHz over the same temperature range. Consistent

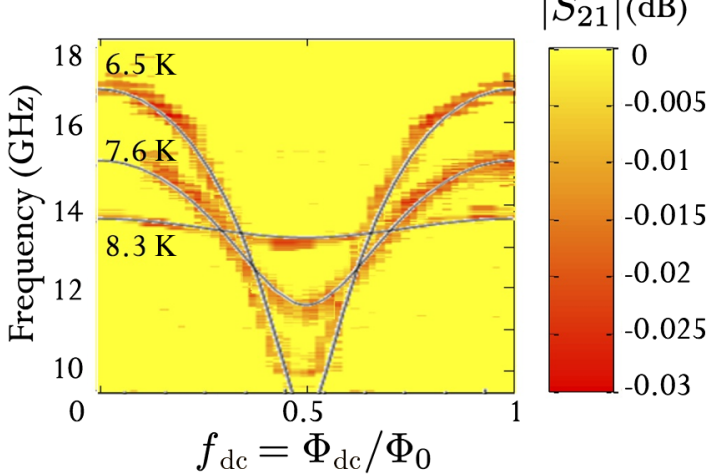

FIG. 3. $\left|S_{21}\right|$ of a single rf SQUID meta-atom as a function of frequency and applied dc flux at three temperatures 6.5, 7.6, and $8.3 \mathrm{~K}$ and $-80-\mathrm{dBm}$ rf power. The resonant response is identified by the red features. The solid lines are the resonant frequency calculated by Eqs. (1), (2), and (8).

with the model, the resonant frequency saturates in the high temperature limit at $f_{0, \text { geo }}=13.5 \pm 0.2 \mathrm{GHz}$ [Eq. (11)].

The temperature dependence of dc flux tuning for the $27 \times 27 \mathrm{rf}$ SQUID array is shown in Fig. 4. The resonance (red features) shows good periodicity with dc flux with a maximum of $21.5 \pm 0.2 \mathrm{GHz}$ and a minimum below $15 \mathrm{GHz}$ at $6.5 \mathrm{~K}$ and $-60-\mathrm{dBm}$ rf power. As temperature increases, the flux tunability is reduced from $>6.5 \mathrm{GHz}$ for $6.5 \mathrm{~K}$ to $2.5 \mathrm{GHz}$ at $7.9 \mathrm{~K}$. A flux offset of about $0.1 \Phi_{0}$ shifts the curve along the flux axis without affecting the periodicity. The periodic horizontal features result from standing waves in the system due to an impedance mismatch in the external circuit.

The meta-atoms in the array respond to the tuning parameters coherently, creating a stronger signal and a slightly wider resonance dip compared to a single $\mathrm{rf}$ SQUID. The flux dependence of the array is qualitatively the same as that of a single SQUID. This similarity is

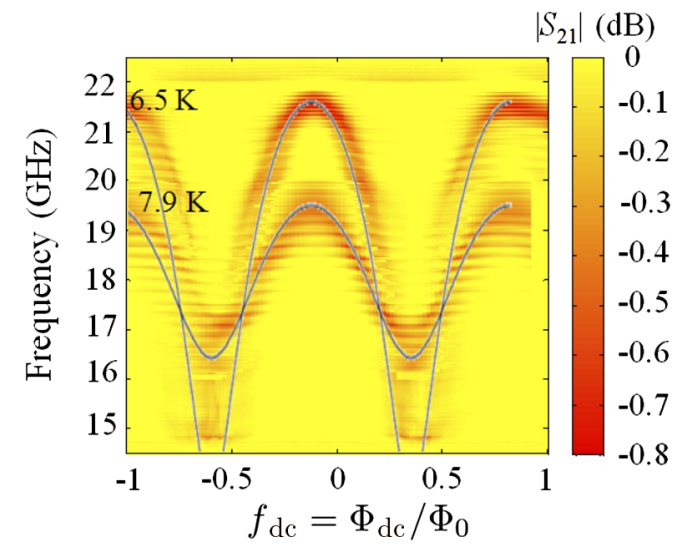

FIG. 4. $\left|S_{21}\right|$ of the $27 \times 27$ rf SQUID array as a function of frequency and applied dc flux at two temperatures 6.5 and $7.9 \mathrm{~K}$ and $-60-\mathrm{dBm}$ rf power. The resonant response is identified by the red features. The solid lines are the resonant frequency calculated for a single SQUID by Eqs. (1), (2), and (8). 
demonstrated by the gray lines in Fig. 4 that are solutions for the resonant frequency of a single SQUID with the following parameters: $L=0.12 \mathrm{nH}$ (nominal value), $C=$ $0.65 \mathrm{pF}, I_{c}(6.5 \mathrm{~K})=1.2 \mu \mathrm{A}$, and $I_{c}(7.9 \mathrm{~K})=0.47 \mu \mathrm{A}$.

The resonance of an $\mathrm{rf}$ SQUID can also be modified by current induced by $\Phi_{\mathrm{rf}}$. Figure 5 shows $\left|S_{21}\right|$ as a function of $\mathrm{rf}$ power and frequency for a single rf SQUID meta-atom in the representative case of $f_{\mathrm{dc}}=1 / 6$ and $T=6.5 \mathrm{~K}$. The resonant response is represented by the red features. When the rf power is low, the resonant frequency remains at a fixed value of $16 \mathrm{GHz}$ and the resonance dip has a constant depth. As the rf power increases, the resonant frequency decreases and the resonance dip becomes shallower. The resonance disappears at intermediate powers before reappearing as a strong resonance at high power $(-57 \mathrm{dBm})$.

Calculating the rf power dependence of the resonance requires a full numerical solution of the nonlinear Eq. (6). Instead of regarding the rf SQUID as an effective medium, we employ Eq. (16) to estimate $\left|S_{21}\right|$. The results of this calculation are plotted as contour lines in Fig. 5, and the calculated minimum of $\left|S_{21}\right|$ is plotted as a dashed line. Both show excellent agreement with the data, including the depth of the $\left|S_{21}\right|$ dip.

There are two transition points in the rf powerdependent resonance at $-75 \mathrm{dBm}\left(\Phi_{\mathrm{rf}} \approx 0.006 \Phi_{0}\right)$ and $-57 \mathrm{dBm}\left(\Phi_{\mathrm{rf}} \approx 0.05 \Phi_{0}\right)$. We can understand the significance of these powers and the behavior in the low, intermediate, and high power regions using the model discussed in Sec. II. In the low power limit, the resonance does not change with increasing $\mathrm{rf}$ power because $S_{21}$ from Eq. (16) does not depend on input rf power $P_{0}$ since $P_{0} \propto V^{2}[V \propto$ $f_{\text {rf }}$ from Eq. (10) and $f_{\mathrm{rf}} \propto \sqrt{P_{0}}$ because of the relationship between input power and magnetic fields in a waveguide].

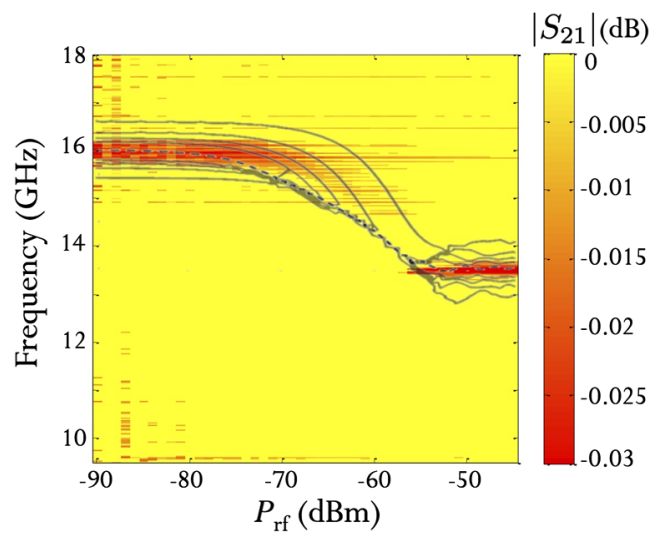

FIG. 5. $\left|S_{21}\right|$ of a single SQUID meta-atom as a function of frequency and $\mathrm{rf}$ power at fixed dc flux $f_{\mathrm{dc}}=1 / 6$ and temperature $T=6.5 \mathrm{~K}$. The resonant response is identified by the red features. The contour lines for $\left|S_{21}\right|$ at $-0.04,-0.03,-0.02$, and $-0.01 \mathrm{~dB}$ are generated by the model; see Eq. (16). The dashed line indicates the model-determined resonant frequency [minimum of $\left.\left|S_{21}(\omega)\right|\right]$.
The first transition occurs at an rf power of $-75 \mathrm{dBm}$, where the resonant frequency begins to decrease and the resonance dip becomes shallower. At this rf power, the oscillation amplitude of $\delta$ during an rf period is $\pi / 2$ on resonance. The resonant frequency is modified because above $-75-\mathrm{dBm}$ rf power, the time-averaged phase difference $\langle\delta\rangle$ deviates from $\delta_{\mathrm{dc}}$. In the case of $f_{\mathrm{dc}}=1 / 6,\langle\delta\rangle>$ $\delta_{\mathrm{dc}}$, resulting in a decrease in $\cos \delta$ from its low power value, a corresponding increase in $L_{\mathrm{JJ}}$ [Eq. (1)], and an ultimate decrease in resonant frequency $f_{0}$ [Eq. (2)]. However, in the case of $f_{\mathrm{dc}}=1 / 2$, the modification in $\langle\delta\rangle$ causes an increase in $\cos \delta$ from its low power value and an ultimate increase in resonant frequency $f_{0}$ that is consistent with the experimental results (not shown). The resonance dip becomes shallower because $V$ is no longer proportional to $f_{\text {rf }}$; instead, the input power $P_{0}$ increases more than the dissipated power $V^{2} / R$.

The second transition occurs at an rf power of $-57 \mathrm{dBm}$, where $\delta$ oscillates with an amplitude exceeding $2 \pi$ on resonance and the Josephson junction undergoes multiple phase slips in each rf period. Above $-57 \mathrm{dBm}$, the SQUID is in the high rf power limit [Eq. (13)], where the tunable resonant frequency reduces to a fixed value [Eq. (11)], i.e., $f_{0 \text {,geo }}=13.5 \mathrm{GHz}$. We expect the losses to be greater in this regime because the phase slips are dissipative, resulting in a deeper resonance dip, which is clearly evident in the data.

A similar evolution of a transition frequency with $\mathrm{rf}$ power has been observed in single qubits coupled to microwave cavities containing a small number of photons [83,84]. The Jaynes-Cummings Hamiltonian shows such behavior at a high photon excitation number, reproducing the observed dispersion and amplitude variation of the transition with increasing of power [85].

\section{DISCUSSION}

The design parameters of the SQUID meta-atom are the critical current $I_{c}(T)$, the capacitance $C$, the subgap resistance $R$, and the loop inductance $L$. In our comparison of data and model for the single meta-atom, we use the nominal design value for loop inductance because the lithographic process dictates the loop geometry to high precision. We adjust the capacitance $C$ and critical current $I_{c}(6.5 \mathrm{~K})$ from their nominal design values to fit the experimentally observed resonant frequencies at $P=$ $-80 \mathrm{dBm}$ using Eq. (2) and $P=-50 \mathrm{dBm}$ using Eq. (11) for $T=6.5 \mathrm{~K}$ and $\Phi_{\mathrm{dc}}=0$. The fit value for capacitance is $30 \%$ higher than the design value, which exceeds the $20 \%$ tolerance quoted for the $\mathrm{Nb} / \mathrm{AlO}_{x} / \mathrm{Nb}$ process [86]. [The same method is used to determine $I_{c}$ $(6.5 \mathrm{~K}), I_{c}(7.9 \mathrm{~K})$, and $C$ for the fit to the array data in Fig. 4.] The subgap resistance $R=820 \Omega$ and the filling fraction $F$ and medium length $l$ in Eqs. (14) and (15) are adjusted to match the width and depth of the observed $\left|S_{21}(\omega)\right|$ minimum at $\Phi_{\mathrm{dc}}=0, T=6.5 \mathrm{~K}$, and $-80 \mathrm{dBm}$. 


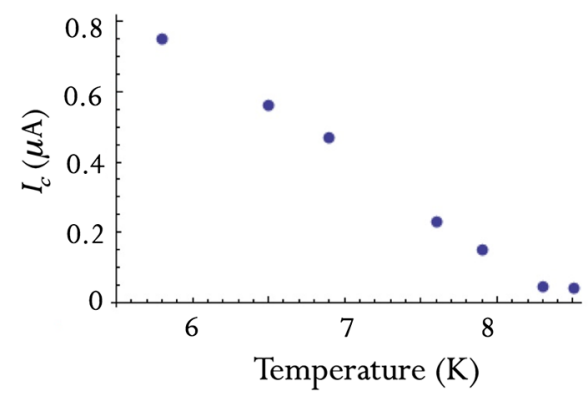

FIG. 6. Critical current temperature dependence $I_{c}(T)$ calculated from measured resonant frequencies for a single SQUID at zero dc flux and corresponding fixed temperatures.

With these parameters, we quantitatively explain the dc flux and rf power dependence of the rf SQUID meta-atom.

We extract the temperature dependence of the critical current $I_{c}(T)$ from the flux dependence of the resonant frequency at different fixed temperatures. The critical current of the junction at each temperature is calculated by substituting the maximum resonant frequency as a function of flux into Eqs. (1) and (2). The results for $I_{c}(T)$, shown in Fig. 6, are consistent with previous results on $\mathrm{Nb} / \mathrm{AlO}_{x} / \mathrm{Al} / \mathrm{Nb}$ tunnel junctions [87].

We have demonstrated tunability of the rf SQUID metaatom with temperature, rf current, and dc magnetic flux. The SQUID meta-atom has an unusual response to changes in temperature. The resonant frequency can either increase or decrease, depending on the dc magnetic flux applied to the meta-atom; the slope of temperature tuning of $f_{0}$ can be large or small and either positive or negative.

Tuning with rf power can also either increase or decrease the resonant frequency, depending on the applied dc magnetic flux. However, the strength of the resonance response varies with rf power while it remains constant with temperature tuning. At low powers $(<-75 \mathrm{dBm})$ and high powers $(>-57 \mathrm{dBm})$, the meta-atom has a resonant interaction with electromagnetic radiation, but at intermediate powers, it becomes essentially transparent. Similar demonstrations of metamaterial-induced transparency have created a tunable "spectral hole" by interfering resonant processes in two or more meta-atoms making up a metamolecule [88-91]. There, the transparency condition in the superconducting metamolecule could be suppressed in a switching transition at high power [36]. In our case, the transparency process is due to the nonlinear Josephson effect and is self-induced, making transparency in the rf SQUID meta-atom simpler than previous implementations. Also unlike other realizations, the transparency condition arises from a decrease in dissipation without enhancement in loss at nearby frequencies [92]. As such, it is potentially useful as a power limiter for sensitive front-end receivers [93].

The dc flux tuning of the SQUID meta-atom is remarkably sensitive. At its maximum value, the flux tunability (defined as the frequency change divided by the change in magnetic field) is approximately $80 \mathrm{THz} / \mathrm{G}$, at $12 \mathrm{GHz}$ and $6.5 \mathrm{~K}$. Hence, only extremely small magnetic fields are required to make very substantial changes in the properties of the meta-atom.

The next question is how quickly can tuning by each parameter be achieved. The shortest time scale for tuning a superconductor is approximately $\hbar / \Delta \sim 1 \mathrm{ps,} \mathrm{where} \Delta$ is the energy gap. In this case, the $R C$ time constant of $0.3 \mathrm{~ns}$ ( $L / R$ time constant $0.4 \mathrm{ps)}$ imposes an upper limit on the intrinsic switching speed. Tuning with temperature depends on changes to the critical current of the junction and is relatively slow, with typical estimates on the order of $10 \mu \mathrm{s}$ [37]. A pulsed rf power measurement of the SQUID meta-atom is consistent with a response time of less than $500 \mathrm{~ns}$. Hence, the rf power tuning time is in the sub- $\mu \mathrm{s}$ range, perhaps only limited by the $R C$ time. Flux tuning of SQUID-like superconducting qubits has been accomplished on nanosecond time scales limited only by the rise time of the applied current pulse [94,95].

The tunability of the meta-atom in normalized units (defined as the tuning frequency range divided by the center frequency of tuning) is $56 \%$. The meta-atom loss varies by about $64 \%$ (defined as the loss range divided by the maximum loss) over this entire range. We estimate the figure of merit (defined as the ratio of the real part of $\mu$ to the imaginary part) to be 1700 on resonance in the case of low power and zero dc flux. The goal of achieving wide meta-atom tunability on short time scales with low loss is well within reach.

The coherent response of the meta-atoms in the $27 \times 27$ array demonstrates the feasibility of a widely tunable $\mathrm{rf}$ SQUID metamaterial. The SQUIDs are close enough that their interactions play a role in their dynamics. We define a coupling parameter that is the ratio of mutual inductance to self-inductance; $\kappa \cong \frac{\mu_{0} \pi r^{4}}{4 d^{3} L}=0.02$, where $r$ is the radius of the loop and $d$ is the center-to-center spacing of the SQUIDs. The ratio of the wavelength to the lattice parameter for the measured array is approximately 350 , which is well within the metamaterial limit. The array displays an overall tunability of $46 \%$ and a flux tunability of $4 \mathrm{THz} / \mathrm{G}$ at $19 \mathrm{GHz}$ and $6.5 \mathrm{~K}$. Future work will further explore arrays of rf SQUID meta-atoms in both the interacting and noninteracting limits.

\section{CONCLUSION}

We have designed, fabricated, and presented an rf superconducting quantum-interference-device meta-atom in a geometry that can be scaled for free-space interactions with electromagnetic fields. The meta-atom proves to be highly tunable with dc magnetic field, rf currents, and temperature. The rf SQUID meta-atom proves to have low losses over its wide (56\%) range of tunability. A novel form of electromagnetically induced transparency has been observed in this meta-atom with increasing induced $\mathrm{rf}$ current. We have obtained a detailed quantitative understanding of this nonlinear meta-atom with first-principles 
modeling. The data and model are in excellent agreement. Finally, a two-dimensional array of rf SQUIDs has shown coherent tuning with dc flux and temperature in a manner similar to a single SQUID.

\section{ACKNOWLEDGMENTS}

This work is supported by the NSF-GOALI Program through Grant No. ECCS-1158644 and the Center for Nanophysics and Advanced Materials (CNAM). Funding for Open Access was provided by the UMD Libraries Open Access Publishing Fund. We thank Masoud Radparvar, Georgy Prokopenko, Jen-Hao Yeh, and Tamin Tai for experimental guidance and helpful suggestions, and Alexey Ustinov, Philipp Jung, and Susanne Butz for helpful discussions. We also thank H. J. Paik and M. V. Moody for use of the pulsed-tube refrigerator. M.T. and D.Z. contributed equally to this work.

[1] V.G. Veselago, The Electrodynamics of Substances with Simultaneously Negative Values of $\epsilon$ and $\mu$, Sov. Phys. Usp. 10, 509 (1968).

[2] D. R. Smith, W. J. Padilla, D. C. Vier, S. C. Nemat-Nasser, and S. Schultz, Composite Medium with Simultaneously Negative Permeability and Permittivity, Phys. Rev. Lett. 84, 4184 (2000).

[3] R. A. Shelby, D. R. Smith, and S. Schultz, Experimental Verification of a Negative Index of Refraction, Science 292, 77 (2001).

[4] J.B. Pendry, Negative Refraction Makes a Perfect Lens, Phys. Rev. Lett. 85, 3966 (2000).

[5] Z. Jacob, L. V. Alekseyev, and E. Narimanov, Optical Hyperlens: Far-Field Imaging beyond the Diffraction Limit, Opt. Express 14, 8247 (2006).

[6] A. Alu and N. Engheta, Pairing an epsilon-Negative Slab with a mu-Negative Slab: Resonance, Tunneling and Transparency, IEEE Trans. Antennas Propag. 51, 2558 (2003).

[7] D. Schurig, J. J. Mock, B. J. Justice, S. A. Cummer, J. B. Pendry, A.F. Starr, and D. R. Smith, Metamaterial Electromagnetic Cloak at Microwave Frequencies, Science 314, 977 (2006).

[8] U. Leonhardt, Optical Conformal Mapping, Science 312, 1777 (2006).

[9] J. B. Pendry, D. Schurig, and D. R. Smith, Controlling Electromagnetic Fields, Science 312, 1780 (2006).

[10] N. I. Landy, S. Sajuyigbe, J. J. Mock, D. R. Smith, and W. J. Padilla, Perfect Metamaterial Absorber, Phys. Rev. Lett. 100, 207402 (2008).

[11] Z. Liu, N. Fang, T.-J. Yen, and X. Zhang, Rapid Growth of Evanescent Wave by a Silver Superlens, Appl. Phys. Lett. 83, 5184 (2003).

[12] R. Ruppin, Surface Polaritons of a Left-Handed Medium, Phys. Lett. A 277, 61 (2000).

[13] R. Ruppin, Surface Polaritons of a Left-Handed Material Slab, J. Phys. Condens. Matter 13, 1811 (2001).
[14] F.D. M. Haldane, Electromagnetic Surface Modes at Interfaces with Negative Refractive Index Make a "NotQuite-Perfect" Lens, arXiv:cond-mat/0206420.

[15] D. R. Smith, D. Schurig, M. Rosenbluth, S. Schultz, S. A. Ramakrishna, and J.B. Pendry, Limitations on Subdiffraction Imaging with a Negative Refractive Index Slab, Appl. Phys. Lett. 82, 1506 (2003).

[16] T. Koschny, R. Moussa, and C. M. Soukoulis, Limits on the Amplification of Evanescent Waves of Left-Handed Materials, J. Opt. Soc. Am. B 23, 485 (2006).

[17] J. O. Dimmock, Losses in Left-Handed Materials, Opt. Express 11, 2397 (2003).

[18] J. Zhou, Th. Koschny, M. Kafesaki, E. N. Economou, J. B. Pendry, and C. M. Soukoulis, Saturation of the Magnetic Response of Split-Ring Resonators at Optical Frequencies, Phys. Rev. Lett. 95, 223902 (2005).

[19] A. Ishikawa, T. Tanaka, and S. Kawata, Negative Magnetic Permeability in the Visible Light Region, Phys. Rev. Lett. 95, 237401 (2005).

[20] Y.A. Urzhumov and G. Shvets, Optical Magnetism and Negative Refraction in Plasmonic Metamaterials, Solid State Commun. 146, 208 (2008).

[21] R. W. Ziolkowski and A. D. Kipple, Application of Double Negative Materials to Increase the Power Radiated by Electrically Small Antennas, IEEE Trans. Antennas Propag. 51, 2626 (2003).

[22] R. W. Ziolkowski and A. Erentok, Metamaterial-Based Efficient Electrically Small Antennas, IEEE Trans. Antennas Propag. 54, 2113 (2006).

[23] A. A. Zharov, I. V. Shadrivov, and Y. S. Kivshar, Nonlinear Properties of Left-Handed Metamaterials, Phys. Rev. Lett. 91, 037401 (2003).

[24] E. B. Wikborg, V. K. Semenov, and K. K. Likharev, RSFQ Front-End for a Software Radio Receiver, IEEE Trans. Appl. Supercond. 9, 3615 (1999).

[25] D. K. Brock, O.A. Mukhanov, and J. Rosa, Superconductor Digital rf Development for Software Radio, IEEE Commun. Mag. 39, 174 (2001).

[26] O. A. Mukhanov, D. Kirichenko, I. V. Vernik, T. V. Filippov, A. Kirichenko, R. Webber, V. Dotsenko, A. Talalaevskii, J.C. Tang, A. Sahu, P. Shevchenko, R. Miller, S. B. Kaplan, S. Sarwana, and D. Gupta, Superconductor Digital-rf Receiver Systems, IEICE Trans. Electron. E91-C, 306 (2008).

[27] S. M. Anlage, The Physics and Applications of Superconducting Metamaterials, J. Opt. 13, 024001 (2011).

[28] J. Gittleman, B. Rosenblum, T.E. Seidel, and A. W. Wicklund, Nonlinear Reactance of Superconducting Films, Phys. Rev. 137, A527 (1965).

[29] S. K. Yip and J. A. Sauls, Nonlinear Meissner Effect in CuO Superconductors, Phys. Rev. Lett. 69, 2264 (1992).

[30] M. Ricci, N. Orloff, and S. M. Anlage, Superconducting Metamaterials, Appl. Phys. Lett. 87, 034102 (2005).

[31] M.C. Ricci and S.M. Anlage, Single Superconducting Split-Ring Resonator Electrodynamics, Appl. Phys. Lett. 88, 264102 (2006).

[32] J. Gu, R. Singh, Z. Tian, W. Cao, Q. Xing, M. He, J. W. Zhang, J. Han, H.-T. Chen, and W. Zhang, Terahertz Superconductor Metamaterial, Appl. Phys. Lett. 97, 071102 (2010). 
[33] V. A. Fedotov, A. Tsiatmas, J. H. Shi, R. Buckingham, P. de Groot, Y. Chen, S. Wang, and N.I. Zheludev, Temperature Control of Fano Resonances and Transmission in Superconducting Metamaterials, Opt. Express 18, 9015 (2010).

[34] H.-T. Chen, H. Yang, R. Singh, J. F. O'Hara, A. K. Azad, S. A. Trugman, Q.X. Jia, and A. J. Taylor, Tuning the Resonance in High-Temperature Superconducting Terahertz Metamaterials, Phys. Rev. Lett. 105, 247402 (2010).

[35] J. Wu, B. Jin, Y. Xue, C. Zhang, H. Dai, L. Zhang, C. Cao, L. Kang, W. Xu, J. Chen, and P. Wu, Tuning of Superconducting Niobium Nitride Terahertz Metamaterials, Opt. Express 19, 12021 (2011).

[36] C. Kurter, P. Tassin, A. P. Zhuravel, L. Zhang, T. Koschny, A. V. Ustinov, C.M. Soukoulis, and S.M. Anlage, Switching Nonlinearity in a Superconductor-Enhanced Metamaterial, Appl. Phys. Lett. 100, 121906 (2012).

[37] V. Savinov, V. A. Fedotov, S. M. Anlage, P. A. J. de Groot, and N.I. Zheludev, Modulating Sub-THz Radiation with Current in Superconducting Metamaterial, Phys. Rev. Lett. 109, 243904 (2012).

[38] S. M. Anlage, H. J. Snortland, and M. R. Beasley, A Current Controlled Variable Delay Superconducting Transmission Line, IEEE Trans. Magn. 25, 1388 (1989).

[39] S. Tahara, S. M. Anlage, J. Halbritter, C.-B. Eom, D. K. Fork, T. H. Geballe, and M. R. Beasley, Critical Currents, Pinning, and Edge Barriers in Narrow $\mathrm{YBa}_{2} \mathrm{Cu}_{3} \mathrm{O}_{7-\delta}$ Thin Films, Phys. Rev. B 41, 11203 (1990).

[40] M. C. Ricci, H. Xu, R. Prozorov, A. P. Zhuravel, A. V. Ustinov, and S.M. Anlage, Tunability of Superconducting Metamaterials, IEEE Trans. Appl. Supercond. 17, 918 (2007).

[41] B. Jin, C. Zhang, S. Engelbrecht, A. Pimenov, J. Wu, Q. $\mathrm{Xu}, \mathrm{C}$. Cao, J. Chen, W. Xu, L. Kang, and P. Wu, Low Loss and Magnetic Field-Tunable Superconducting Terahertz Metamaterial, Opt. Express 18, 17504 (2010).

[42] A.P. Zhuravel, S.M. Anlage, and A. V. Ustinov, Microwave Current Imaging in Passive HTS Components by Low-Temperature Laser Scanning Microscopy (LTLSM), J. Supercond. Novel Magn. 19, 625 (2006).

[43] A.P. Zhuravel, A. G. Sivakov, O. G. Turutanov, A. N. Omelyanchouk, S. M. Anlage, A. Lukashenko, A. V. Ustinov, and D. Abraimov, Laser Scanning Microscopy of HTS Films and Devices (Review Article), Low Temp. Phys. 32, 592 (2006).

[44] C. Kurter, A. P. Zhuravel, J. Abrahams, C. L. Bennett, A. V. Ustinov, and S.M. Anlage, Superconducting rf Metamaterials Made with Magnetically Active Planar Spirals, IEEE Trans. Appl. Supercond. 21, 709 (2011).

[45] C. Kurter, A. P. Zhuravel, A. V. Ustinov, and S. M. Anlage, Microscopic Examination of Hot Spots Giving Rise to Nonlinearity in Superconducting Resonators, Phys. Rev. B 84, 104515 (2011).

[46] A. P. Zhuravel, C. Kurter, A. V. Ustinov, and S. M. Anlage, Unconventional rf Photoresponse from a Superconducting Spiral Resonator, Phys. Rev. B 85, 134535 (2012).

[47] V. Savinov, A. Tsiatmas, A. R. Buckingham, V. A. Fedotov, P.A.J. de Groot, and N. I. Zheludev, Flux
Exclusion Superconducting Quantum Metamaterial: Towards Quantum-Level Switching, Sci. Rep. 2, 450 (2012).

[48] M. Tinkham, Introduction to Superconductivity (McGrawHill, New York, 1996), 2nd ed.

[49] T. P. Orlando and K. A. Delin, Foundations of Applied Superconductivity (Addison-Wesley, Reading, MA, 1991).

[50] K. K. Likharev, Dynamics of Josephson Junctions and Circuits (Gordon and Breach, New York, 1986).

[51] F. Auracher and T. Van Duzer, $r f$ Impedance of Superconducting Weak Links, J. Appl. Phys. 44, 848 (1973).

[52] H. Salehi, A. H. Majedi, and R. R. Mansour, Analysis and Design of Superconducting Left-Handed Transmission Lines, IEEE Trans. Appl. Supercond. 15, 996 (2005).

[53] H. Salehi, R. R. Mansour, and A. H. Majedi, Nonlinear Josephson Left-Handed Transmission Lines, IET Microwaves Antennas Propag. 1, 69 (2007).

[54] A. H. Silver and J.E. Zimmerman, Quantum States and Transitions in Weakly Connected Superconducting Rings, Phys. Rev. 157, 317 (1967).

[55] B. Chesca, M. Muck, and Y. Zhang, Microwave Superconductivity (Kluwer, Dordrecht, 2001).

[56] C. Du, H. Chen, and S. Li, Quantum Left-Handed Metamaterial from Superconducting QuantumInterference Devices, Phys. Rev. B 74, 113105 (2006).

[57] C. Du, H. Chen, and S. Li, Stable and Bistable SQUID Metamaterials, J. Phys. Condens. Matter 20, 345220 (2008).

[58] N. Lazarides and G.P. Tsironis, rf Superconducting Quantum Interference Device Metamaterials, Appl. Phys. Lett. 90, 163501 (2007).

[59] A. I. Maimistov and I. R. Gabitov, Nonlinear Response of a Thin Metamaterial Film Containing Josephson Junctions, Opt. Commun. 283, 1633 (2010).

[60] N. Lazarides and G.P. Tsironis, Multistability and SelfOrganization in Disordered SQUID Metamaterials, Supercond. Sci. Technol. 26, 084006 (2013).

[61] A. L. Rakhmanov, A. M. Zagoskin, S. Savel'ev, and F. Nori, Quantum Metamaterials: Electromagnetic Waves in a Josephson Qubit Line, Phys. Rev. B 77, 144507 (2008).

[62] M. A. Castellanos-Beltran and K.W. Lehnert, Widely Tunable Parametric Amplifier Based on a Superconducting Quantum Interference Device Array Resonator, Appl. Phys. Lett. 91, 083509 (2007).

[63] M. A. Castellanos-Beltran, K. D. Irwin, G. C. Hilton, L. R. Vale, and K. W. Lehnert, Amplification and Squeezing of Quantum Noise with a Tunable Josephson Metamaterial, Nat. Phys. 4, 929 (2008).

[64] E. A. Ovchinnikova, S. Butz, P. Jung, V. P. Koshelets, L. V. Filippenko, A. S. Averkin, S. V. Shitov, and A. V. Ustinov, Design and Experimental Study of Superconducting LeftHanded Transmission Lines with Tunable Dispersion, Supercond. Sci. Technol. 26, 114003 (2013).

[65] P. Jung, S. Butz, S. V. Shitov, and A. V. Ustinov, Low-Loss Tunable Metamaterials Using Superconducting Circuits with Josephson Junctions, Appl. Phys. Lett. 102, 062601 (2013).

[66] S. Butz, P. Jung, L. V. Filippenko, V. P. Koshelets, and A. V. Ustinov, Protecting SQUID Metamaterials against 
Stray Magnetic Fields, Supercond. Sci. Technol. 26, 094003 (2013).

[67] S. Butz, P. Jung, L. V. Filippenko, V.P. Koshelets, and A. V. Ustinov, A One-Dimensional Tunable Magnetic Metamaterial, Opt. Express 21, 22540 (2013).

[68] J. Luine, L. Abelson, D. Brundrett, J. Burch, E. Dantsker, K. Hummer, G. Kerber, M. Wire, K. Yokoyama, D. Bowling, M. Neel, S. Hubbell, and K. Li, Application of a dc SQUID Array Amplifier to an Electrically Small Active Antenna, IEEE Trans. Appl. Supercond. 9, 4141 (1999).

[69] P. Caputo, J. Tomes, J. Oppenlander, Ch. Haussler, T. Trauble, and N. Schopohl, Two-Tone Response in Superconducting Quantum Interference Filters, IEEE Trans. Appl. Supercond. 17, 722 (2007).

[70] V. Kornev, I. Soloviev, N. Klenov, A. Sharafiev, and O. Mukhanov, Array Designs for Active Electrically Small Superconductive Antennas, Physica (Amsterdam) 479C, 119 (2012).

[71] V. K. Kornev, I. I. Soloviev, A. V. Sharafiev, N. V. Klenov, and O. A. Mukhanov, Active Electrically Small Antenna Based on Superconducting Quantum Array, IEEE Trans. Appl. Supercond. 23, 1800405 (2013).

[72] O. V. Snigirev, M. L. Chukharkin, A. S. Kalabukhov, M. A. Tarasov, A. A. Deleniv, O. A. Mukhanov, and D. Winkler, Superconducting Quantum Interference Filters as rf Amplifiers, IEEE Trans. Appl. Supercond. 17, 718 (2007).

[73] V. K. Kornev, I. I. Soloviev, N. V. Klenov, and O. A. Mukhanov, High-Linearity SQIF-like Josephson-Junction Structures, IEEE Trans. Appl. Supercond. 19, 741 (2009).

[74] J. T. Jeng, H. C. Hung, C. R. Lin, C. H. Wu, K. L. Chen, J. C. Chen, H. C. Yang, S. H. Liao, and H. E. Horng, Fluxto-Voltage Transfer Function of the Series-SQUID Array with Grain-Boundary Josephson Junctions, IEEE Trans. Appl. Supercond. 15, 793 (2005).

[75] A. C. Bruno, M. A. Espy, D. D. Clark, A. N. Matlashov, and R. H. Kraus, Jr., Digital Spatial Filter Made from a SQUID Array, IEEE Trans. Appl. Supercond. 13, 779 (2003).

[76] J. Oppenlander, Ch. Haussler, T. Trauble, P. Caputo, J. Tomes, A. Friesch, and N. Schopohl, Two Dimensional Superconducting Quantum Interference Filters, IEEE Trans. Appl. Supercond. 13, 771 (2003).

[77] C. M. Falco, Phase-Space of a Driven, Damped Pendulum (Josephson Weak Link), Am. J. Phys. 44, 733 (1976).

[78] B. Chesca, Theory of $r f$ SQUIDs Operating in the Presence of Large Thermal Fluctuations, J. Low Temp. Phys. 110, 963 (1998).

[79] S. Bermon and T. Gheewala, Moat-Guarded Josephson SQUIDs, IEEE Trans. Magn. 19, 1160 (1983).

[80] Whiteley Research, Inc., http://www.wrcad.com/.
[81] D. R. Smith and J.B. Pendry, Homogenization of Metamaterials by Field Averaging, J. Opt. Soc. Am. B 23, 391 (2006).

[82] S. Doyle, Ph.D. thesis, University of Cardiff, 2008.

[83] M. D. Reed, L. DiCarlo, B. R. Johnson, L. Sun, D. I. Schuster, L. Frunzio, and R. J. Schoelkopf, High-Fidelity Readout in Circuit Quantum Electrodynamics Using the Jaynes-Cummings Nonlinearity, Phys. Rev. Lett. 105, 173601 (2010).

[84] S. Novikov, J.E. Robinson, Z. K. Keane, B. Suri, F. C. Wellstood, and B.S. Palmer, Autler-Townes Splitting in a Three-Dimensional Transmon Superconducting Qubit, Phys. Rev. B 88, 060503(R) (2013).

[85] L. S. Bishop, E. Ginossar, and S. M. Girvin, Response of the Strongly Driven Jaynes-Cummings Oscillator, Phys. Rev. Lett. 105, 100505 (2010).

[86] Hypres, Niobium Integrated Circuit Fabrication Process No. 03-10-45 Design Rules, http://www.hypres.com/ foundry/niobium-process/.

[87] I. P. Nevirkovets, J. B. Ketterson, and J. M. Rowell, Modified Superconductor-Insulator-Normal MetalInsulator-Superconductor Josephson Junctions with High Critical Parameters, J. Appl. Phys. 89, 3980 (2001).

[88] V. A. Fedotov, M. Rose, S. L. Prosvirnin, N. Papasimakis, and N. I. Zheludev, Sharp Trapped-Mode Resonances in Planar Metamaterials with a Broken Structural Symmetry, Phys. Rev. Lett. 99, 147401 (2007).

[89] N. Papasimakis, V. A. Fedotov, N. I. Zheludev, and S. L. Prosvirnin, Metamaterial Analog of Electromagnetically Induced Transparency, Phys. Rev. Lett. 101, 253903 (2008).

[90] P. Tassin, L. Zhang, Th. Koschny, E. N. Economou, and C. M. Soukoulis, Low-Loss Metamaterials Based on Classical Electromagnetically Induced Transparency, Phys. Rev. Lett. 102, 053901 (2009).

[91] C. Kurter, P. Tassin, L. Zhang, T. Koschny, A. P. Zhuravel, A. V. Ustinov, S.M. Anlage, and C. M. Soukoulis, Classical Analogue of Electromagnetically Induced Transparency with a Metal-Superconductor Hybrid Metamaterial, Phys. Rev. Lett. 107, 043901 (2011).

[92] A. A. Abdumalikov, Jr., O. Astafiev, A. M. Zagoskin, Yu. A. Pashkin, Y. Nakamura, and J.S. Tsai, Electromagnetically Induced Transparency on a Single Artificial Atom, Phys. Rev. Lett. 104, 193601 (2010).

[93] O. A. Mukhanov, D. Gupta, A. M. Kadin, and V. K. Semenov, Superconductor Analog-to-Digital Converters, Proc. IEEE 92, 1564 (2004).

[94] F. G. Paauw, A. Fedorov, C. J. P. M. Harmans, and J. E. Mooij, Tuning the Gap of a Superconducting Flux Qubit, Phys. Rev. Lett. 102, 090501 (2009).

[95] X. Zhu, A. Kemp, S. Saito, and K. Semba, Coherent Operation of a Gap-Tunable Flux Qubit, Appl. Phys. Lett. 97, 102503 (2010). 